\title{
Einsatz von Informationstechnologien zur Bewältigung der Dokumentations- und Informationsbedürfnisse im Rahmen der medizinischen Leistungserbringung
}

\author{
Notwendigkeit standardisierter elektronischer Austauschformate \\ S. Hölzer ${ }^{a, b, c}$, R. Schweiger ${ }^{b}$, J. Dudeck ${ }^{b, c}$
}

a H+ Die Spitäler der Schweiz.

b Institut für Medizinische Informatik der Justus-LiebigUniversität Giessen und HL7 User Group Deutschland.

c Working Group II des European Committee for Standardization (CEN/TC 251)

Korrespondenz: Dr. Simon Hölzer $\mathrm{H}+$ Die Spitäler der Schweiz Lorrainestrasse $4 \mathrm{~A}$ Postfach 302

CH-3000 Bern

Tel. 0313351124

Fax 0313351170

E-Mail: simon.hoelzer@hplus.ch
Die gesetzlichen Auflagen im Rahmen der Medizinischen Statistik in der Schweiz und die Einführung von Patientenklassifikationssystemen führen zu einem Zwang einer möglichst vollständigen und medizinisch plausiblen Dokumentation von individuellen Erkrankungen und den erbrachten medizinischen Versorgungsleistungen und stellen die Leistungserbringer vielfach vor eine in der Praxis noch ungenügend gelöste Herausforderung [1, 2].

\section{Dokumentation und Datenintegration}

So wird seitens der Dokumentation insbesondere im Hinblick auf zugrundeliegende Diagnosen, Komorbiditäten, Komplikationen und medizinische Interventionen ein konsistenter Gebrauch verfügbarer Klassifikationen (z.B. International Statistical Classification of Diseases and Related Health Problems [ICD-10] und Schweizerische Operationsklassifikation [CHOP]) gefordert [2-4]. Dies hat zur Folge, dass digitale Versionen in den Informationssystemen vorgehalten oder direkt integriert sind. Hier werden hohe Anforderungen an die Pflege und insbesondere das Management unterschiedlicher bzw. neuer Versionen dieser Standardterminologien gestellt. Diese Anforderungen machen ein einheitliches Format für die Speicherung der Inhalte notwendig, auf welches system- und herstellerunabhängig zugegriffen werden kann. Diese einheitlichen Formate sind derzeit noch eher die Seltenheit, was die Integration dieser Quellen in bestehende Krankenhaus- und Informationssystemen erschwert.

Auf der anderen Seite müssen administrative und klinische Informationen aus unterschiedlichen operationalen Systemen (Administration, Abteilungssysteme, Funktionsbereiche usw.) übergreifend integriert werden und für die Codierung zur Verfügung stehen [5]. Auch an dieser Stelle benötigt man von technischer Seite ein Austauschformat, dessen Inhalte und Strukturen definiert werden können, ohne auf proprietäre Lösungen zurückgreifen zu müssen, um eine Kommunikation herzustellen.

Für die effiziente Verwirklichung dieser Aufgaben bietet es sich an, auf ein gemeinsames Format eines Datenstandards zurückzugreifen. In den vergangenen zweieinhalb Jahren hat sich hierfür auf internationaler Ebene als technische Lösung für verschiedene Anwendungsgebiete die sogenannte eXtensible Markup Language (XML) herauskristallisiert. XML hat sich in kurzer Zeit als Format zur Übertragung strukturierter Dokumente basierend auf Technologien des Internet etabliert [6, 7]. Im Gegensatz zu den gängigen Internetseiten werden hier nicht primär Informationen zur Darstellung eines Dokumentes hinterlegt, sondern die Textinhalte können bezeichnet (Patientenname, Geburtsdatum, Hauptdiagnose, Nebendiagnose usw.), hierarchisch strukturiert und untereinander verknüpft sein, so dass ein gezieltes Auffinden und Darstellen (z.B. als Tabelle von Patienten mit bestimmten Haupt- und Nebendiagnosen nach Alter geordnet) ermöglicht wird. Das Ablegen von Information beruht hier auf einem dokumentenorientierten Ansatz, welcher sich im Vergleich zu einer Datenbank durch seine Flexibilität bei der Verarbeitung von strukturierten (z. B. Tabellenwerk der ICD-10, Band 1) und teilstrukturierten Informationen (z.B. ärztlicher Austrittsbericht, Pathologiebericht, Röntgenanforderung usw.) hervorhebt. Jedoch müssen die Bedeutung dieser Inhalte und deren Beziehungen zueinander transparent festgelegt und vereinheitlicht werden. 


\section{Standardisierung}

Diese Aufgaben werden für die Medizin international von Standardisierungsgremien wie dem «European Committee for Standardization» (CEN/TC 251) und der «Health Level 7 Organisation» (HL7) übernommen [8]. Auch in der Schweiz hat sich eine aktive HL7 User Group etabliert, welche eng mit der deutschen User Group zusammenarbeitet und in der ein Grossteil der nationalen IT-Anbieter vertreten sind. XML wird von HL7 (www.hl7.org) für unterschiedliche Zwecke, aber insbesondere zur Kommunikation im Gesundheitswesen verstärkt eingesetzt. So versucht man im Rahmen der «Clinical Document Architecture» (CDA), basierend auf XML, ein Gerüst für den Austausch von Dokumenten innerhalb und zwischen Institutionen im Gesundheitswesen zu schaffen. Diese Architektur erlaubt es klinische Dokumente auszutauschen, indem deren Struktur und - in benötigtem Umfang - deren Inhalte definiert werden. Jedes dieser Dokumente kann für sich alleinstehend Informationen $\mathrm{zu}$ einem Patienten, inklusive Text, Bild, Ton und andere multimediale Inhalte, umfassen. Dieser Ansatz vereinfacht den Austausch von Informationen zwischen unterschiedlichen Institutionen im Gesundheitswesen (ambulanter und stationärer Sektor) durch die Möglichkeit der direkten elektronischen Verarbeitung bei gleichzeitigem Erhalt der Lesbarkeit (Darstellung) der Originaldokumente [9].

\section{Entscheidungsunterstützung}

Stehen auf der einen Seite das Zusammenführen der administrativen und klinischen Daten und die Codierung nach standardisierten Vokabularen, kann auf der anderen Seite durch einen dokumentenorientierten Ansatz mit XML eine direkte Unterstützung des ärztlichen und pflegerischen Management erfolgen:

Zunehmend werden Versorgungsstandard über medizinische Leitlinien definiert. Diese verstehen sich als ein Werkzeug zur Implementierung evidenzbasierter Medizin und werden zumeist von den medizinischen Fachgesellschaften in Form von Textdokumenten entwickelt. Leitlinien und im engeren Sinne «Clinical Pathways» enthalten Informationen bzw. die medizinische Rationale zur «ausreichenden, zweckmässigen und wirtschaftlichen» Versorgung des individuellen Patienten [10]. Es bietet sich an, diese Dokumente in XML aufzubereiten und zu pflegen, um sie einer Weiterverarbeitung, z.B. zur Entscheidungsunterstützung am Arztarbeitsplatz, zugänglich zu machen (Beispielanwendung unter www.patientcare.de). Dabei werden Wissensinhalte strukturiert und insbesondere deren klinische Relevanz im Hinblick auf bestimmte Patientengruppen (Alter, Geschlecht, Haupt-, Nebendiagnosen usw.) und Erkrankungscharakteristika (Stadium, Schweregrad usw.) in Beziehung gesetzt. Dieses Vorgehen erlaubt eine Kopplung von Informationen aus der elektronischen Patientenakte, z. B. über Diagnose- oder Prozedurencodes, mit den korrespondierenden Wissensressourcen. Dadurch lässt sich ein kontextsensitiver Zugriff auf vorhandenes bzw. benötigtes Wissen in einer spezifischen Entscheidungssituation realisieren [11, 12]. Diese Infrastruktur lässt sich als Steuerungsinstrument im Rahmen von Disease-Management-Programmen oder den in der Schweiz entwickelten Integrierten Patientenpfaden nutzen.

So kann eine Kopplung von Codes innerhalb der elektronischen Patientenakte mit standardisierten Patientenpfaden erfolgen, welche wiederum in XML gepflegt werden und damit einen kontextsensitiven Zugriff auf deren Inhalte zulassen. Damit ermöglicht die geforderte zeitnahe Erfassung relevanter Patientendaten notwendige klinische Analysen dieser Informationen bzw. die elektronische Weiterverarbeitung z.B. in Form von entscheidungsunterstützenden Funktionalitäten. Im Hinblick auf den durch die Einführung von Patientenklassifikationssystemen erzwungenen Dokumentationsaufwand beim Nachweis medizinischer Leistungen und bei den zugrundeliegenden Diagnosen (einschliesslich Schweregrad, Begleiterkrankungen, Komplikationen usw.) kann eine Infrastruktur basierend auf oben genannten Konzepten den Workflow und die Arbeitsbelastung günstig beeinflussen.

Nur so lassen sich langfristig die vorhanden personellen Ressourcen für die eigentlichen ärztlichen und pflegerischen Aufgaben, insbesondere die Arbeit am Patienten, einsetzen und unter dem steigenden Kostendruck eine qualitativ hochwertige Medizin weiterführen. Anwendungen auf der Basis von XML in der Medizin [13] werden die Arbeit der Endbenutzer in vielfältiger Weise erleichtern, wenn entsprechende benutzerfreundliche Applikationen zur Verfügung stehen. 


\section{Literatur}

1 Cimino JJ. Terminology tools: state of the art and practical lessons. Methods Inf Med 2001;40(4): 298-306.

2 Schrappe M. [Quality management within the requirements of current health care policy; introduction - political and institutional aspects]. Med Klin 2001;96(7):417-23.

3 Schopen M. [The implementation of the International Classification of Diseases (ICD-10) in Germany. Tools and information on the internet] Anaesthesist 1999;48(12):924-30.

4 Stausberg J, Zaiss A, Fuchs J, Berke A. Using ICD10 for case groups. Stud Health Technol Inform 2000;77:359-63.

5 Cimino JJ, Socratous SA, Clayton PD. Internet as clinical information system: application development using the World Wide Web. J Am Med Inform Assoc 1995;2(5):273-84.

6 Schweiger R, Burkle T, Holzer S, Dudeck J. XML structured clinical information: a practical example. Stud Health Technol Inform 2000;77: 822-6.

7 URL: http://www.w3.org/XML/ (last visited 18.11.2001).
8 URL: http://www.hl7.org/ (last visited 18.11.2001).

9 Dolin RH, Alschuler L, Beebe C, Biron PV, Boyer SL, Essin D, et al. The HL7 Clinical Document Architecture. J Am Med Inform Assoc 2001;8(6):552-69.

10 Grimshaw JM, Russell IT. Effect of clinical guidelines on medical practice: a systematic review of rigorous evaluations. Lancet 1993;342(8883): 1317-22.

11 Holzer S, Schweiger RK, Dudeck J. [Current approaches for the implementation of guidelines by means of information technologies]. Z Arztl Fortbild Qualitatssich 2001;95(8):555-9.

12 Hoelzer S, Schweiger RK, Boettcher HA, Tafazzoli AG, Dudeck J. Value of XML in the implementation of clinical practice guidelines the issue of content retrieval and presentation. Med Inform Internet Med 2001;26(2):131-46.

13 Schweiger R, Hoelzer S, Altmann U, Rieger J, Dudeck J. Plug-and-play XML: a health care perspective. J Am Med Inform Assoc 2002;9(1): 37-48. 